\title{
New model-based
}

SFB

823

\section{bioequivalence statistical}

approaches for

pharmacokinetic studies with

sparse sampling

Florence Loingeville, Julie Bertrand,

Thu Thuy Nguyen, Satish Sharan, Kairui Feng,

Wanjie Sun, Jing Han, Stella Grosser,

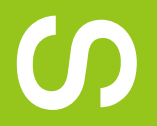

Liang Zhao, Lanyan Fang, Kathrin Möllenhoff, Holger Dette, France Mentré

Nr. 19/2020

$\mathcal{O}$

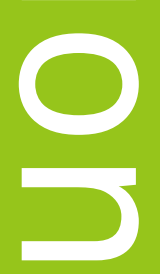

0

(1)

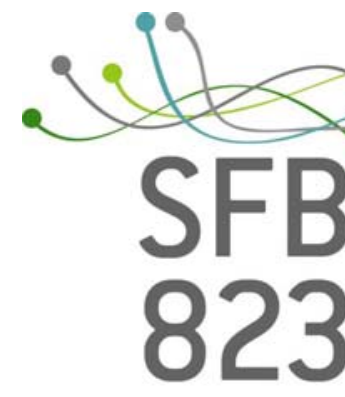

(1) 



\title{
New model-based bioequivalence statistical approaches for pharmacokinetic studies with sparse sampling
}

\author{
Florence Loingeville ${ }^{1,2}$, Julie Bertrand ${ }^{1}$, Thu Thuy Nguyen ${ }^{1}$, \\ Satish Sharan ${ }^{3}$, Kairui Feng ${ }^{3}$, Wanjie Sun ${ }^{4}$, Jing $\mathrm{Han}^{4}$, \\ Stella Grosser ${ }^{4}$, Liang Zhao ${ }^{3}$, Lanyan Fang $^{3}$, \\ Kathrin Möllenhoff ${ }^{5,6}$, Holger Dette ${ }^{5}$, France Mentré ${ }^{1}$ \\ ${ }^{1}$ University of Paris, IAME INSERM, UMR 1137, 75018 Paris, France \\ ${ }^{2}$ Univ. Lille, CHU Lille, ULR 2694 - METRICS : Evaluation of Health Technologies \\ and Medical Practices, F-59000 Lille, France
}

${ }^{3}$ Division of Quantitative Methods and Modeling, Office of Research Standards, Office of Generic Drugs, Center for Drug Evaluation and Research, Food and Drug Administration, Silver Spring MD 20993, USA

${ }^{4}$ Office of Biostatistics, Office of Translational Sciences, Center for Drug Evaluation and Research, Food and Drug Administration, Silver Spring MD 20993, USA

${ }^{5}$ Department of Mathematics, Ruhr-Universitat Bochum, Germany

${ }^{6}$ Institute of Medical Statistics and Computational Biology, Faculty of Medicine, University of Cologne, Cologne, Germany

\footnotetext{
${ }^{2}$ Corresponding author: florence.loingeville@univ-lille.fr; Laboratoire de biomathématiques, Faculté de Pharmacie, 3 Rue du Professeur Laguesse, 59000 Lille, France
} 


\begin{abstract}
1
Introduction: In traditional pharmacokinetic (PK) bioequivalence analysis, two one-sided 2 tests (TOST) are conducted on the area under the concentration-time curve and the max- 3 imal concentration derived using a non-compartmental approach. When rich sampling is ${ }_{4}$ unfeasible, a model-based (MB) approach, using nonlinear mixed effect models (NLMEM) is possible. However, MB-TOST using asymptotic standard errors (SE) presents increased 6 type I error when asymptotic conditions do not hold.

Methods : In this work, we propose three alternative calculations of the SE based on i) an adaptation to NLMEM of the correction proposed by Gallant, ii) the a posteriori distribution of the treatment coefficient using the Hamiltonian Monte Carlo algorithm, and iii) parametric random effects and residual errors bootstrap. We evaluate these approaches by simulations, for two-arms parallel and two-periods two-sequences cross-over design with rich $(n=10)$ and sparse $(n=3)$ sampling under the null and the alternative hypotheses, with MB-TOST.

Results: All new approaches correct for the inflation of MB-TOST type I error in PK studies with sparse designs. The approach based on the a posteriori distribution appears to be the best compromise between controlled type I errors and computing times.

Conclusion: MB-TOST using non-asymptotic SE controls type I error rate better than when using asymptotic SE estimates for bioequivalence on PK studies with sparse sampling.

Keywords and Phrases: pharmacokinetics, bioequivalence, nonlinear mixed effects model, two ${ }_{21}^{21}$ one-sided tests, non-asymptotic standard error 


\section{Introduction}

Bioequivalence studies are routinely conducted for the development of generics or the adoption 24 of new formulations of existing drug. According to current guidelines by regulation authorities 25 both in the US and the EU $[1,2]$, bioequivalence between a reference (R) and a test (T) product ${ }^{26}$ is to be assessed based on the comparison of their respective area under the time-concentration curves (AUC) and maximal concentrations $\left(\mathrm{C}_{\max }\right)$. The presently recommended statistical approach is to claim bioequivalence if the boundaries of the 90\%-confidence intervals around the ratios of $\mathrm{AUC}$ and $\mathrm{C}_{\max }$ geometric means of both groups do fall between 0.8 and 1.25. This is equivalent to performing a two one-sided tests (TOST) proposed by Schuirmann [3].

Traditionally, individual estimates of AUC are obtained using non-compartmental analysis (NCA-TOST). Based on few hypotheses, NCA requires dense pharmacokinetic (PK) sampling. In especially fragile populations (e.g., children or patients), or for specific indications (e.g., ophthalmic drugs), it may be challenging and/or unethical to collect such dense sampling.

Therefore to assess the PK bioequivalence of two ophthalmic drugs on a study with only onetime point per subject, Shen et al. proposed a non parametric bootstrap NCA-based TOST[4]. A population PK model-based (MB) approach is another appealing alternative when dense PK samplings cannot be obtained, as it lowers the individual sampling burden by borrowing information across patients. .

In 2010, Dubois et al. compared the type I error and power of the NCA-TOST to a TOST ${ }^{41}$ based on individual empirical Bayes estimates (EBE) from a nonlinear mixed effect model ${ }_{42}$ (NLMEM)[5]. They found that, when the shrinkage is above 20\%, using NCA TOST leads to a modestly increased type I error whereas using TOST on EBE leads to a more severe type I error inflation. They suggested to perform a TOST directly on the treatment effect estimate from the NLMEM (MB-TOST) using the asymptotic standard error (SE). In 2011, they evaluated the MB-TOST using Wald test and likelihood ratio test and found an inflation of the type I error when asymptotic conditions are not met, which is the underlying condition for applying Delta method, that is, for very sparse sample (number of samples per subject is limited), or small sample size (number of subjects is small), or high variability. Further, they associated this inflation to an under-estimation of the SE of the treatment effect coefficient, due to the use of an asymptotic approximation, i.e., the observed Fisher Information matrix (FIM). So the MBTOST in its current form does not meet the standards of regulatory agencies for confirmatory tests.

Therefore, the primary objective of this work is to propose alternative approaches to calculate the SE, guarantying for the MB-TOST a nominal type I error on sparse sampling PK studies. First, we adapted the correction based on the work by Gallant [6] which Bertrand et al. extended to Wald tests in NLMEM, in case of small sample size studies [7]. Second, we proposed to sample 
in the a posteriori distribution of population parameters obtained by Hamiltonian Monte-Carlo $\quad 59$ using Stan [8], as proposed by Ueckert et al. [9]. Third, we used parametric bootstrap, which 60 was shown to perform better than case bootstrap and non parametric residual bootstrap when ${ }_{61}$ the true model and variance distribution are used [10].

We evaluated MB-TOST using these approaches by clinical trial simulations with parallel and ${ }_{63}$ cross-over designs, with rich and sparse samplings.

Although the TOST is very efficient in most cases, it has proven to be too conservative on drugs with high variability [11]. Therefore, Möllenhoff et al. proposed a bioequivalence optimal test (BOT) as an alternative to the TOST for bioequivalence assessment in such situations [12]. They adapted this test to the MB approach (MB-BOT), and showed that this method appears to have closer type I errors to the conventionally accepted significance level of 0.05 than the MB-TOST for drugs with high variability. However, they also noticed an inflation of the type I errors on sparse designs, showing that the SE-computation method is also an issue with MB-BOT. In supplementary material 2, we evaluate MB-BOT along with the proposed SEcalculation approaches. Then, we further study the conjoint influence of design and variability on the SE of the treatment effect on AUC and $C_{m a x}$, and thus on type I error and power of MB tests. Thereafter, we determine a threshold on the SE above which MB-BOT should be recommended over MB-TOST.

In Section 2, we introduce the NLMEM, the MB-TOST as well as the different SE calculations. $\quad 78$ In Section 3, we present the clinical trial simulations performed to evaluate the approaches. In 79 Section 4, we present the results, i.e., type I error and power of the different approaches and 80 finally in Section 5, we discuss the conclusions and perspectives of this work.

\section{Methods}

\subsection{Nonlinear mixed effects models}

The concentration $y_{i, j, k}$ of subject $i(i=1, \ldots, N)$, at period $\mathrm{k}(k=1,2)$, at sampling time ${ }^{84}$ $t_{i, j, k}\left(j=1, \ldots, n_{i}\right)$ is described by a nonlinear function $f$ depending on the vector of individual 85 parameters $\phi_{i, k}$ of subject $i$ at period $k$

$$
y_{i, j, k}=f\left(t_{i, j, k}, \phi_{i, k}\right)+g\left(t_{i, j, k}, \phi_{i, k}\right) \varepsilon_{i, j, k} .
$$

The $l^{\text {th }}$ individual parameter $\phi_{i, k, l}(l=(1, \ldots, p))$ is defined by the following equation, where $p \quad{ }_{87}$ is the number of PK parameters

$$
\log \left(\phi_{i, k, l}\right)=\log \left(\lambda_{l}\right)+\beta_{l}^{T r} T r_{i, k}+\beta_{l}^{P} P_{k}+\beta_{l}^{S} S_{i}+\eta_{i, l}+\kappa_{i, k, l}
$$


with $\lambda_{l}$ the $l^{\text {th }}$ element of the vector of fixed effects for the covariate reference class. $\operatorname{Tr}_{i, k}, P_{k}, \quad{ }_{89}$ $S_{i}$ are known vectors of, respectively, the treatment, the period, and the sequence covariates. 90 $\beta_{l}^{T r}, \beta_{l}^{P}$, and $\beta_{l}^{S}$ are the $l^{t h}$ elements of the vectors of coefficients of the treatment, the period, 91 and the sequence effects for the individual parameter. 92

$\eta_{i, l}$ is the $l^{\text {th }}$ element of the vector $\eta_{i}$ of random effects of subject $i$ capturing the between-subject ${ }_{93}$ variability $(\mathrm{BSV}) . \kappa_{i, k, l}$ is the $l^{\text {th }}$ element of the vector $\kappa_{i, k}$ of random effects of subject $i$ at 94 period $k$ capturing the within-subject variability (WSV). $\eta_{i}$ and $\kappa_{i, k}$ are assumed independent 95 and normally distributed with zero mean and covariance matrix, respectively $\Omega$ and $\Gamma$, both 96 of size $p \times p$. We define $\omega_{l}^{2}$ the between-subject variance of the $l^{\text {th }}$ parameter, and $\gamma_{l}^{2}$ the ${ }_{97}$ within-subject variance of the $l^{\text {th }}$ parameter. $\quad 98$ The residual errors $\varepsilon_{i, j, k}$ are supposed independent and identically distributed according to a 99 normal centered distribution with variance 1 . The error model can be additive $g\left(t_{i, j, k}, \phi_{i, k}\right)=a, \quad 100$ proportionnal $g\left(t_{i, j, k}, \phi_{i, k}\right)=b \times f\left(t_{i, j, k}, \phi_{i, k}\right)$, i.e., additive on log-concentrations, or combined 101 $g\left(t_{i, j, k}, \phi_{i, k}\right)=a+b \times f\left(t_{i, j, k}, \phi_{i, k}\right) . \quad 102$

We denote by $\theta=\left(\lambda, \beta^{T r}, \beta^{P}, \beta^{S}, \Omega, \Gamma, a, b\right)$ the vector of all parameters of the model, and by 104 $\widehat{V A R}(\hat{\theta})$ the estimation variance-covariance matrix, derived as the inverse of the observed FIM. ${ }_{105}$

Here, bioequivalence is assessed on $\beta_{S P}^{T r}$ the coefficient of the treatment on the secondary PK ${ }_{107}$ parameters of interest $\mathrm{SP}=\left\{A U C\right.$ or $\left.C_{\max }\right\}$. For each secondary parameter, $\beta_{S P}^{T r}$ is a function ${ }_{108}$ of $\lambda$ and $\beta^{T r}$ and its SE is derived from $\widehat{V A R}(\hat{\theta})$.

\subsection{Model-based TOST}

The MB-TOST global null hypothesis is expressed as $H_{0}: \beta_{S P}^{T r} \leq-\delta$ or $\beta_{S P}^{T r} \geq \delta$ and can be ${ }_{111}$ divided in two sub-hypotheses: $H_{0,-\delta}: \beta_{S P}^{T r} \leq-\delta$ and $H_{0, \delta}: \beta_{S P}^{T r} \geq \delta$. 112 Therefore, the MB-TOST consists in two Wald statistics: $W_{-\delta}=\left(\hat{\beta}_{S P}^{T r}+\delta\right) / S E\left(\beta_{S P}^{T r}\right)$ and 113 $W_{\delta}=\left(\hat{\beta}_{S P}^{T r}-\delta\right) / S E\left(\beta_{S P}^{T r}\right)$, respectively testing $H_{0,-\delta}$ and $H_{0, \delta}$, with $S E\left(\beta_{S P}^{T r}\right)$ the standard error ${ }_{114}$ on the estimation of a secondary parameter $S P=A U C, C_{\max }$. In an asymptotic setting, $W_{-\delta} \quad 115$ and $W_{\delta}$ can be assumed to follow a Gaussian distribution under $H_{0,-\delta}$ and $H_{0, \delta}$, respectively. $\quad 116$ So, the global null hypothesis $H_{0}$ is rejected with type I error $\alpha$ if $W_{-\delta} \geq z_{1-\alpha}$ and $W_{\delta} \leq-z_{1-\alpha} \quad{ }_{117}$ where $z_{1-\alpha}$ is the $(1-\alpha)$-quantile of the standard normal distribution. 118 Alternatively, one can compute the $(1-2 \alpha)$ confidence interval (CI) of $\beta_{S P}^{T r}$ and reject the global ${ }_{119}$ null hypothesis if it is included in the interval $[-\delta ; \delta] . \quad 120$

For MB-TOST, the asymptotic approach (Asympt.) consists in using $\widehat{V A R}(\hat{\theta}), \hat{\lambda}$, and $\beta^{\hat{T} r}{ }_{122}$ for deriving the $\mathrm{SE}$ of the secondary parameters $\left(\mathrm{SE}\left(\beta_{S P}^{T r}\right)\right)$ with the delta method [13]. The ${ }_{123}$ analytical formulas are shown in detail in Appendix A of Dubois et al. [14]. ${ }^{124}$ 


\subsection{New approaches for standard error (SE) calculations}

Gallant. This method consists in multiplying the asymptotic SE by a factor equal to $\sqrt{\frac{n_{P} \times N}{d f_{G}}} \quad{ }_{126}$ where $n_{P}$ is the number of periods, $\mathrm{N}$ is the number of subjects, and $d f_{G}=n_{P} \times N-p$. For ${ }_{127}$ MB-TOST, the reference distribution is the Student distribution.

Sampling in the a posteriori distribution (Post). This method consists in sampling in ${ }_{129}$ the a posteriori distribution of $\beta^{T r}$. The a posteriori distribution is obtained using Hamiltonian 130 Monte-Carlo (HMC). We assigned default priors to the fixed effects $\left(\lambda, \beta^{T r}, \beta^{P}, \beta^{S}\right)$ and non- ${ }_{131}$ informative half Cauchy priors to variance terms. The HMC chain was initialized at $\hat{\theta}, \hat{\eta}_{i}$, and ${ }_{132}$ $\hat{\kappa}_{i, k}$ from the NLMEM analysis using the SAEM algorithm. For each resulting sample of $\lambda_{133}$ and $\beta^{T r}$, we derive a corresponding $\beta_{S P}^{T r}$ and the standard deviation of this series is the Post ${ }_{134}$ $S E\left(\beta_{S P}^{T r}\right)$.

Parametric random effect and residual bootstrap (Boot). This method consists in 136 simulating $b=1, \ldots, B$ datasets with the original bioequivalence study design. The subject ${ }_{137}$ random effects and residuals are issued from distributions with means and variances equal to 138 the estimated population parameters from the original bioequivalence study NLMEM analysis. 139 Then, the B datasets are fitted with a NLMEM and B replicates of $\beta_{S P_{b}}^{\hat{T} r}$ are calculated as ${ }_{140}$ functions of the $\hat{\lambda_{b}}$ and $\beta_{b}^{\hat{T} r}$ estimates. The standard deviation of this series is the $\operatorname{Boot} S E\left(\beta_{S P}^{T r}\right) . \quad{ }_{141}$

\section{Simulation Study}

\subsection{Pharmacokinetic model}

We used the PK model from Dubois et al. [14], which describes concentrations of the anti- ${ }^{144}$ asthmatic drug theophylline, for both reference and test group, with a one-compartment distribution (apparent volume, $\mathrm{V} / \mathrm{F}$ ) and first-order absorption (absorption rate, Ka) and elimination (apparent clearance, CL/F). We fixed the dose to $D=4 \mathrm{mg}$ for all subjects.

For the reference treatment, we considered $\lambda_{K a}=1.50 / \mathrm{h}, \lambda_{C L / F}=40.00 \mathrm{~mL} / \mathrm{h}$, and $\lambda_{V / F}=0.50 \mathrm{~L} . \quad 148$ We considered a combined error model with $a=0.1 \mathrm{mg} / \mathrm{L}$ and $\mathrm{b}=10 \%$, corresponding to a low ${ }_{149}$ residual variability.

The bioequivalence threshold $\delta$ was set at $\log (1.25) \approx 0.22$ as recommended by the guidelines [15]. 


\subsection{Treatment effect}

We simulated under one null hypothesis $H_{0, \log (0.8)}$, i.e., $\log \left(A U C^{T} / A U C^{R}\right)=\log \left(C_{\max }^{T} / C_{\max }^{R}\right)={ }_{155}$ $\log (0.8)$, where $A U C^{T} / A U C^{R}$ and $C_{\max }^{T} / C_{\max }^{R}$ are the ratios of geometric means of $\mathrm{T}$ to $\mathrm{R}$ for- ${ }^{156}$ mulations of AUC and $C_{\max }$ respectively [16]. The corresponding treatment effect coefficients ${ }_{157}$ modifying both $C L / F$ and $V / F$ are $\beta_{V}^{T r}=\beta_{C L}^{T r}=\log (1.25) . \quad 158$ We also simulated under one alternative hypothesis by setting $\beta_{C L}^{T r}=\beta_{V}^{T r}=\log (1)$, which ${ }^{159}$ corresponds to $\beta_{A U C}^{T r}=\beta_{C_{\max }}^{T r}=\log (1)=0$

\subsection{Study Design}

We simulated two-arms parallel and 2-periods 2-sequences cross-over designs (as in [14]). For ${ }_{163}$ both trials, we simulated a rich and a sparse design, both with $\mathrm{N}=40$ subjects. For the rich design, there were $\mathrm{n}=10$ samples per subject, taken at $0.25,0.5,1,2,3.5,5,7,9,12,24$ hours after dosing. For the sparse design, we simulated $n=3$ samples per subject, taken at $0.25,3.35$ and 24 hours after dosing. We considered the same sparse design as in $[14,17]$. The sampling times for this design were chosen by maximization of the determinant of the Fisher information matrix for an individual nonlinear model using the fixed effect values. This was done using the PFIM software [18] with a sampling window from 15 min to 24 hours.

We first simulated a parallel design (Figure 1 ), where $N / 2$ subjects receive the reference treatment (R) whereas the other $N / 2$ subjects are allocated to the test treatment $(\mathrm{T})$. Such a design is often chosen to assess the bioequivalence of drugs with long half-lives preventing the use of each patient as his own control within the time constraints of drug development. We simulated BSV random effects with $\omega_{K a}=\omega_{C L / F}=22 \%$ and $\omega_{V / F}=11 \%$, i.e., rather low BSV. For parallel trials, the period effects $\beta_{l}^{P}$, the sequence effects $\beta_{l}^{S}$, and the WSV $\kappa_{i, k, l}$ in the expression of the log of individual parameters (2) are null.

We also simulated a two-periods, two-sequences cross-over design (Figure 2), which is the gold- ${ }_{178}$ standard in bioequivalence trials. In these trials, the $N / 2$ subjects of the first sequence $\left(S_{1}\right) \quad 179$ receive the reference $(\mathrm{R})$ treatment at period $1\left(P_{1}\right)$, and the test $(\mathrm{T})$ treatment at period 2180 $\left(P_{2}\right)$, whereas the $N / 2$ subjects of the second sequence $\left(S_{2}\right)$ receive treatments in the reverse order. We simulated BSV and WSV random effects with $\omega_{K a}=\omega_{C L / F}=\omega_{V / F}=50 \%$, and $\gamma_{K a}=\gamma_{C L / F}=\gamma_{V / F}=15 \%$, i.e., rather high BSV and rather low WSV.

\subsection{Implementation and evaluation}

We evaluated the type I error and power of the MB-TOST on $\beta_{A U C}^{T r}$ and $\beta_{C \max }^{T r}$ using the dif- ${ }_{185}$ ferent approaches, at the nominal level $\alpha=5 \%$ on the different scenarios. 
Five hundred data sets were simulated per scenario, using the $\mathrm{R}$ software.

The parameters of the NLMEM were estimated using the SAEM algorithm. For the parallel design, we used the $\mathrm{R}$ package saemix version 1.2 [19], and for the crossover design, we used the monolix software version 2018R2 [20].

We used the same parameterisation with both softwares; 10 Monte Carlo Markov chains, 300 iterations in the exploratory phase, and 100 iterations in the smoothing phase.

For the Asympt approach, the FIM was obtained by linearisation.

For the Post approach, we used the Rstan package with 1000 iterations and 100 burn-in, so ${ }_{196}$ that we obtained 900 samples.

For the Boot approach, we simulated $B=250$ data sets.

All calculations were run on an i7-5600 U CPU computer, with frequency $2.60 \mathrm{GHz}, 4$ cores, 8200 GB of RAM.

\section{Results}

\subsection{Two-arms parallel}

For both the sparse and rich designs, the Relative Biais (RBiais) and the Relative Root Mean 204 Square Errors (RRMSE) were below $10 \%$ for the fixed effects and close to 0 for the treatment ${ }^{205}$ effect coefficients (Table I in supplementary material 1). For the BSV and residual error stan- ${ }^{206}$ dard deviations, there was a upward trend in the RBiais, below $10 \%$ for the rich design and 207 up to $30 \%$ for the sparse design. The RRMSE also increased for the sparse design, up to $121 \% \quad 208$ for the additive residual error standard deviation. Yet, there was no major concern with the 209 estimation of the NLMEM parameters. 210

On the rich design, MB-TOST conserved a nominal type I error with all different SE calculations ${ }_{211}$ (Figure 3). On the sparse design with $\mathrm{N}=40$, MB-TOST for $\beta_{A U C}^{T r}$ using the asymptotic SE led ${ }_{212}$ to an inflated type I error (Figure 3). However, using the alternative calculations of the SE, ${ }^{213}$ the inflation was corrected.

In supplementary material 2, we evaluate the proposed approaches to compute the SE along 215 with both MB-TOST and MB-BOT on a sparser design (with N=12 subjects). Then, we ${ }_{216}$ further explore the relationship between $S E\left(\beta_{S P}^{T r}\right)$ and the MB-TOST type I error and derive ${ }^{217}$ a critical threshold when MB-TOST no longer controls its nominal level and MB-BOT should ${ }_{218}$ be used instead. 
The simulated power of MB-TOST using the different SE calculations were of similar order and 220 of reasonable size $>70 \%$ (Table I). We observe higher power to conclude to bioequivalence on ${ }^{221}$ $C_{\max }$ compared to $A U C$ as we simulated $\omega_{C \max }=10 \%$ and $\omega_{A U C}=22 \%$, respectively. ${ }_{222}$ With regard to computational times, Asympt and Gallant approaches took a few seconds per ${ }_{223}$ data set, whereas running Post took a few minutes, and Boot close to 1 hour. In fact, we did ${ }_{224}$ not compute the bootstrap-based SE on the sparse design with $\mathrm{N}=20$ (scenario simulated under ${ }^{225}$ the null only), given its computational burden and the good performances of the Gallant and ${ }_{226}$ Post alternatives.

\subsection{Two-periods, two-sequences cross-over}

The Rbias and RRMSE on all parameters for the scenario with a sparse design, which we ${ }^{229}$ expected to be the most challenging under the null, are listed in Supplementary Material 1, 230 Table II. For the fixed effects and treatment effect coefficients, all Rbias were below 5\% and ${ }_{231}$ the RRMSE were below 25\%. For the BSV, WSV and residual error standard deviations, the Rbias showed a downward trend (but for $\gamma_{K a}$ ), and the RRMSE were below 40\%. Again, there was no major concern with the estimation of the NLMEM parameters.

MB-TOST for $\beta_{A U C}^{T r}$ controlled its nominal level using all SE calculation whatever the design ${ }^{235}$ (Figure 4). Whereas MB-TOST for $\beta_{C \max }^{T r}$ using the asymptotic SE obtained an inflation of ${ }^{236}$ the type I error at $7.6 \%$ on the rich design and $7.8 \%$ on the sparse design. This inflation was ${ }^{237}$ corrected using all the alternative SE calculations. Given its computational burden and the ${ }_{238}$ good performance of other alternative SE calculations, we only evaluated the bootstrap-based ${ }_{239}$ SE on the most challenging design, i.e., the sparse design where it took about 5 hours per data 240 set.

MB-TOST for $\beta_{A U C}^{T r}$ and $\beta_{C \max }^{T r}$ obtained extremely high power (>95\%) using all SE calculations whatever the design (Table II).

\section{Discussion}

In this work we proposed, and evaluated by simulations, three alternative SE calculations to 246 correct for the type I error inflation of MB-TOST in PK bioequivalence studies with sparse ${ }_{247}$ sampling. MB-TOST using the three alternative SE calculations provided both a controlled ${ }_{248}$ nominal type I error and satisfactory power, on parallel and cross-over studies with rich and 249 sparse sampling. Here, we used $\mathrm{B}=250$ iterations for bootstrap. This relative small number 250 nonetheless enabled the bootstrap approach to provide a controlled type I error. However, 251 its computational burden proved particularly limiting, especially given the good performances 252 of the SE calculations based on the work of Gallant [6] and the a posteriori distribution [9]. ${ }^{253}$ 
The latter calculation is particularly appealing given the SE calculation based on the work of ${ }^{254}$ Gallant will, by design, be of limited interest for high N. For now, it requires calling the Stan 255 software and further work is needed to embed this calculation within the monolix software or ${ }_{256}$ saemix R package.

Besides sparse sampling, products with high PK variability present another methodological 258 challenge in bioequivalence studies. Already, Haidar et al. proposed a scaling approach setting 259 a constraint on the geometric mean ratio [21] and the US Food and Drug Administration's 260 Office of Generic Drugs developed a reference-scaled average bioequivalence approach [22]. In 261 [12], Möllenhoff et al. proposed a new test, the MB-BOT, more appropriate for drugs with high ${ }^{262}$ variability, when the sample size is not large enough. In Supplementary Material 2, we showed ${ }^{263}$ that MB-BOT can also benefit from alternative SE calculations in PK BE studies with sparse ${ }^{264}$ sampling. Further, we derived a threshold for the treatment effect SE above which MB-BOT ${ }_{265}$ should be prefered to MB-TOST.

One limitation of the present work is the number of simulated datasets for each scenario un- ${ }^{267}$ der consideration. We choose to simulate 500 data sets because of the computational burden ${ }^{268}$ and because we could effectively compare the approaches in term of type 1 error. Another ${ }_{269}$ non-negligible limitation is the use of the simulated model to perform the MB-TOST. Indeed, 270 we did not investigate the robustness to model misspecification, or consider a model averaging 271 approach [23]. However we reckon that when bioequivalence studies are performed, there exists 272 some accumulated knowledge on the drug PK model (resulting from either a bottom-up or a ${ }_{273}$ top-down approach), at least in the reference treatment group. 274

Finally, statistical methods have recently been proposed to control the nominal type I error in 276 bioequivalence studies using adaptive designs [24]. This methods rely on an adaptation of the 277 NCA-TOST and we believe there is a case for exploring these methods using MB-TOST, and 278 non-asymptotic SE for PK bioequivalence studies with sparse sampling. 279

\section{Conclusion}

We recommend to use non-asymptotic SE, based on the a posteriori distribution of the treat- ${ }^{282}$ ment effect coefficient, to test for bioequivalence on pharmacokinetic studies with sparse sam- ${ }^{283}$ pling with MB-TOST. 


\section{Acknowledgments}

This work was supported by the Food and Drug Administration (FDA) under contract

75F40119C1011. The authors thank FDA for this funding. The views in this paper do not ${ }^{287}$ necessarily reflect the views or policies of the FDA. The work of H. Dette was supported in part 288 by the Collaborative Research Center "Statistical modeling of nonlinear dynamic processes" 289 (SFB 823, Teilprojekt T1) of the German Research Foundation (DFG) 


\section{References}

[1] US Department of Health and Human Services and others. FDA Guid- ${ }^{292}$ ance for Industry, Statistical Approaches to Establishing Bioequivalence. ${ }^{293}$ https://wwwfdagov/media/70958/download. 2001;.

[2] EMEA C. Note for Guidance on the Investigation of Bioavailability and Bioequivalence. 295 CPMP/EWP/QWP/1401/98, London; 2001.

[3] Schuirmann DJ. A comparison of the two one-sided tests procedure and the power approach 297 for assessing the equivalence of average bioavailability. Journal of Pharmacokinetics and 298 Biopharmaceutics. 1987;15(6):657-680. 299

[4] Shen M, Machado SG. Bioequivalence evaluation of sparse sampling pharmacokinet- 300 ics data using bootstrap resampling method. Journal of Biopharmaceutical Statistics. 301 $2017 ; 27(2): 257-264 . \quad 302$

[5] Dubois A, Gsteiger S, Pigeolet E, Mentré F. Bioequivalence tests based on individual esti- ${ }^{303}$ mates using non-compartmental or model-based analyses: evaluation of estimates of sample ${ }^{304}$ means and type I error for different designs. Pharmaceutical Research. 2010;27(1):92-104. 305

[6] Gallant AR. Seemingly unrelated nonlinear regressions. Journal of Econometrics. 306 $1975 ; 3(1): 35-50$.

[7] Bertrand J, Comets E, Chenel M, Mentré F. Some alternatives to asymptotic tests for 308 the analysis of pharmacogenetic data using nonlinear mixed effects models. Biometrics. 309 2012;68(1):146-155. 310

[8] Stan Development Team. RStan: the R interface to Stan, Version 2.12.0; 2016. http: 311 //mc-stan.org/.

[9] Ueckert S, Riviere MK, Mentre F. Improved Confidence Intervals and P-Values by Sam- 313 pling from the Normalized Likelihood. In: Journal of Pharmacokinetics and Pharmacody- 314 namics. vol. 42; 2015. p. S56-S57.

[10] Thai HT, Mentré F, Holford NH, Veyrat-Follet C, Comets E. A comparison of boot- 316 strap approaches for estimating uncertainty of parameters in linear mixed-effects models. 317 Pharmaceutical Statistics. 2013;12(3):129-140. 318

[11] Tsai CA, Huang CY, Liu Jp. An approximate approach to sample size determination in 319 bioequivalence testing with multiple pharmacokinetic responses. Statistics in Medicine. 320 2014;33(19):3300-3317. 
[12] Möllenhoff K, Loingeville F, Bertrand J, Nguyen TT, Sharan S, Sun G, et al.. Efficient 322 model-based Bioequivalence Testing; arXiv:2002.09316[stat.ME]. 2020.

[13] Oehlert GW. A note on the delta method. The American Statistician. 1992;46(1):27-29. 324

[14] Dubois A, Lavielle M, Gsteiger S, Pigeolet E, Mentré F. Model-based analyses of bioe- 325 quivalence crossover trials using the stochastic approximation expectation maximisation 326 algorithm. Statistics in Medicine. 2011;30(21):2582-2600. 327

[15] FDA. Guidance for industry: statistical approaches to establishing bioequivalence. Center 328 for Drug Evaluation and Research, Food and Drug Administration, Rockville, Maryland. 329 2001;. $\quad 330$

[16] Liu JP, Weng CS. Bias of two one-sided tests procedures in assessment of bioequivalence. 331 Statistics in Medicine. 1995;14(8):853-861. 332

[17] Panhard X, Mentré F. Evaluation by simulation of tests based on non-linear mixed-effects 333 models in pharmacokinetic interaction and bioequivalence cross-over trials. Statistics in 334 medicine. 2005;24(10):1509-1524. 335

[18] Dumont C, Lestini G, Le Nagard H, Mentré F, Comets E, Nguyen TT, et al. PFIM 4.0, 336 an extended $\mathrm{R}$ program for design evaluation and optimization in nonlinear mixed-effect 337 models. Computer methods and programs in biomedicine. 2018;156:217-229. 338

[19] Comets E, Lavenu A, Lavielle M. Parameter estimation in nonlinear mixed effect models 339 using saemix, an R implementation of the SAEM algorithm. Journal of Statistical Software. 340 $2017 ; 80: 1-42$. 341

[20] Monolix version 2018R2 . Antony, France: Lixoft SAS; 2018. http://lixoft.com/ 342 products/monolix/. 343

[21] Haidar SH, Makhlouf F, Schuirmann DJ, Hyslop T, Davit B, Conner D, et al. Evalua- ${ }^{344}$ tion of a scaling approach for the bioequivalence of highly variable drugs. The American 345 Association of Pharmaceutical Scientists Journal. 2008;10(3):450-454. 346

[22] Davit BM, Chen ML, Conner DP, Haidar SH, Kim S, Lee CH, et al. Implementation of a 347 reference-scaled average bioequivalence approach for highly variable generic drug products 348 by the US Food and Drug Administration. The American Association of Pharmaceutical 349 Scientists Journal. 2012;14(4):915-924. 350

[23] Buatois S, Ueckert S, Frey N, Retout S, Mentré F. Comparison of model averaging and 351 model selection in dose finding trials analyzed by nonlinear mixed effect models. The 352 AAPS journal. 2018;20(3):56. 
[24] Maurer W, Jones B, Chen Y. Controlling the type I error rate in two-stage sequen- 354 tial adaptive designs when testing for average bioequivalence. Statistics in Medicine. 355 2018;37(10):1587-1607. 

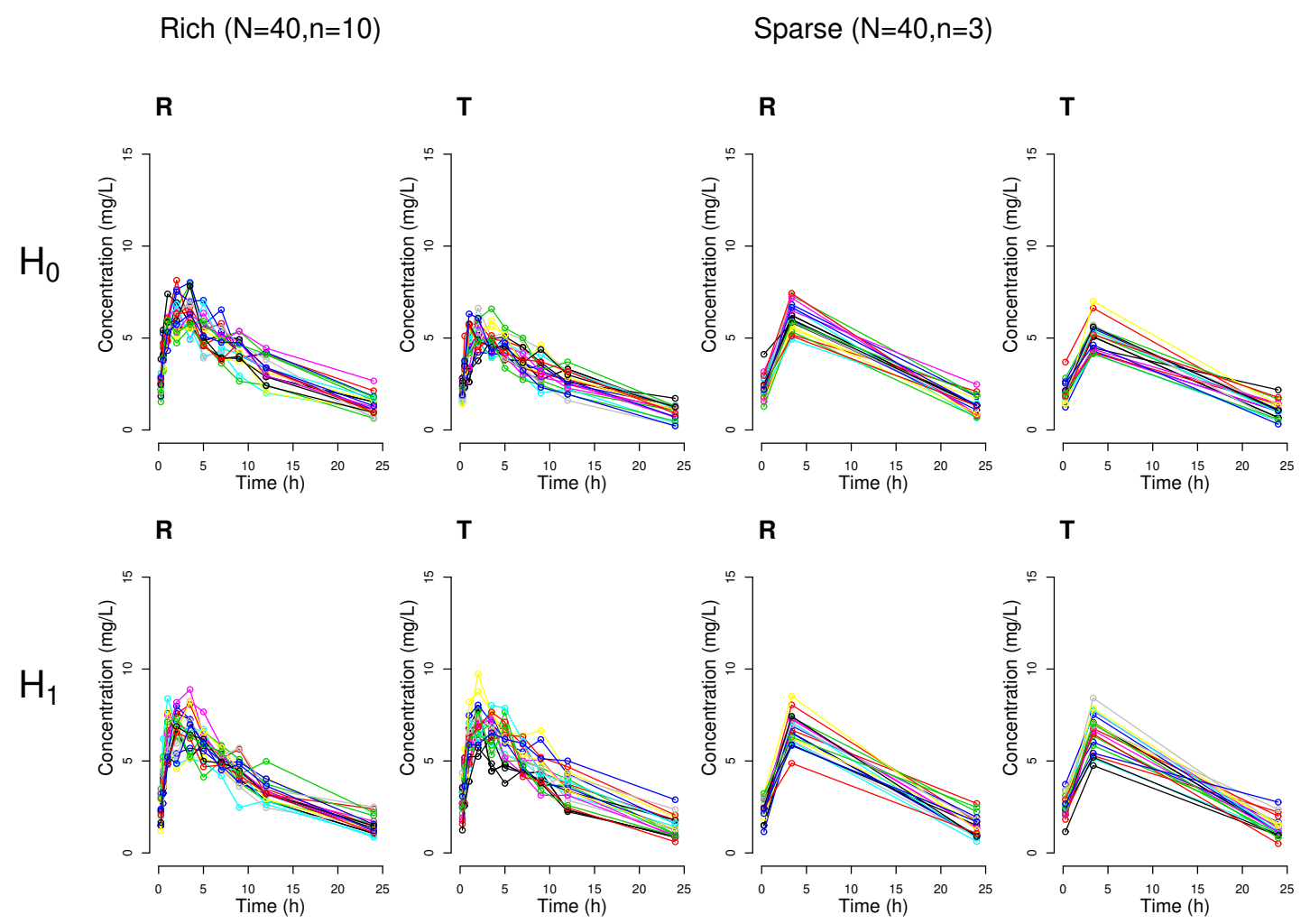

Figure 1: Spaghetti plots of simulated concentrations versus time for the two-arms parallel design under $H_{0}$ (top) and $H_{1}$ (bottom) for rich (columns 1 and 2) and sparse (columns 3 and 4) designs, in the reference (R, columns 1 and 3 ) and the test (T, columns 2 and 4) treatment groups.

Table I: Estimated power of MB-TOST on $\beta_{A U C}^{T r}$ and $\beta_{C \max }^{T r}$, using the different SE calculations, for the parallel rich and sparse designs on the 500 data sets.

\begin{tabular}{|c|c|c||c|c|}
\cline { 2 - 5 } \multicolumn{1}{c|}{} & \multicolumn{2}{c||}{ Rich $(\mathrm{n}=10)$} & \multicolumn{2}{c|}{ Sparse $(\mathrm{n}=3)$} \\
\cline { 2 - 5 } \multicolumn{1}{c|}{} & $\beta_{A U C}^{T r}$ & $\beta_{C \max }^{T r}$ & $\beta_{A U C}^{T r}$ & $\beta_{C \max }^{T r}$ \\
\hline Asympt & 0.830 & 1.000 & 0.804 & 1.000 \\
\hline Gallant & 0.782 & 1.000 & 0.762 & 0.998 \\
\hline Post & 0.772 & 0.966 & 0.712 & 0.990 \\
\hline Boot & 0.832 & 1.000 & 0.800 & 1.000 \\
\hline
\end{tabular}




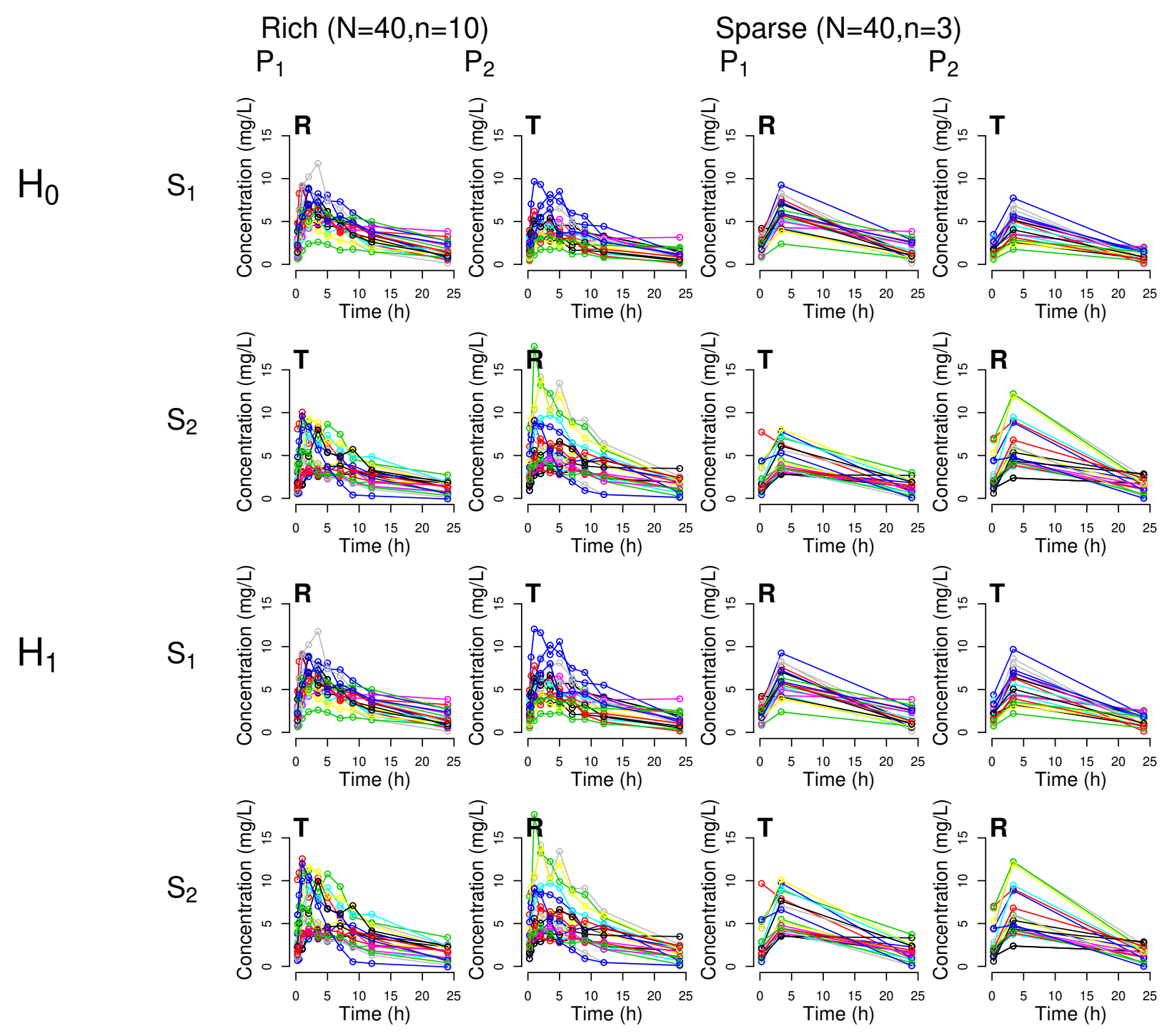

Figure 2: Spaghetti plots of simulated concentrations versus time for the 2-periods 2-sequences $\left(S_{1}, S_{2}\right)$ cross-over design under $H_{0}$ (lines 1 and 2) and under $H_{1}$ (lines 3 and 4) for rich (columns 1 and 2) and sparse (columns 3 and 4$)$ designs, in period $1\left(P_{1}\right.$, columns 1 and 3 ) and period $2\left(P_{2}\right.$, columns 2 and 4$)$. 

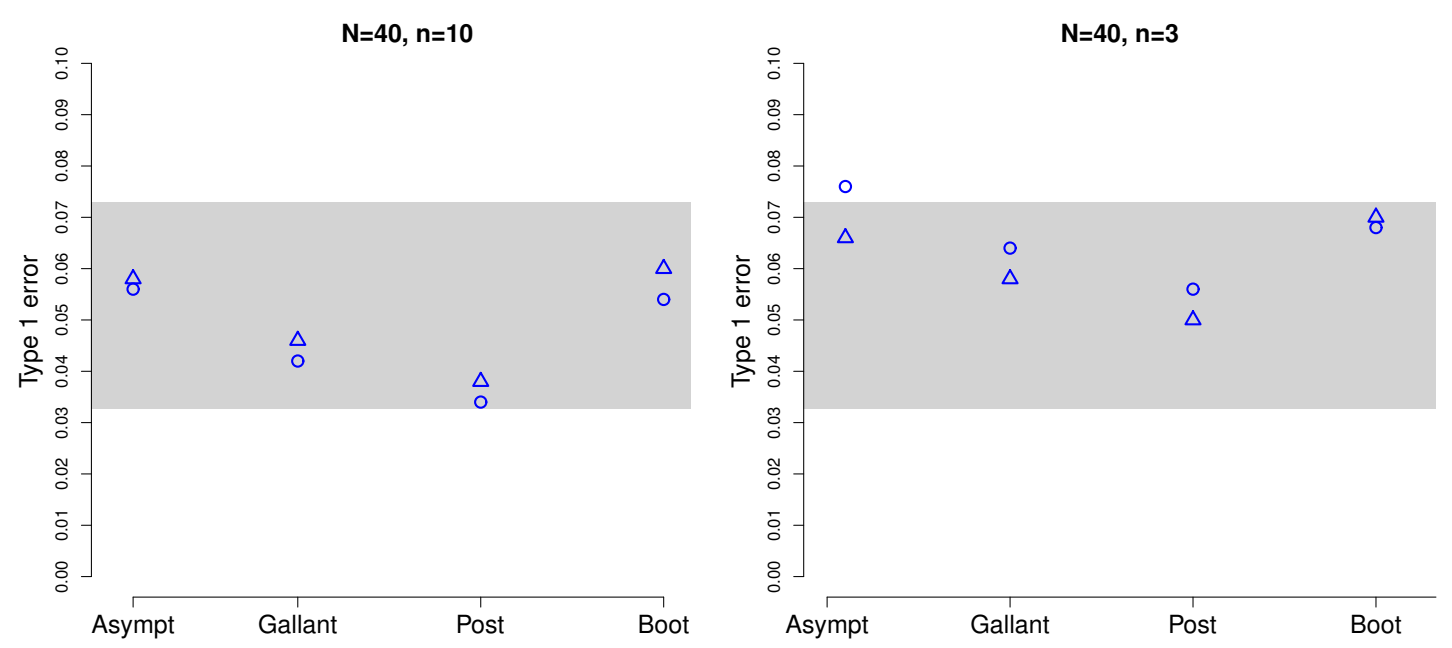

Figure 3: Type I errors of MB-TOST on $\beta_{A U C}^{T r}(\mathrm{o})$ and on $\beta_{C \max }^{T r}(\triangle)$ using the different SE calculations on the parallel rich (left), and sparse (right) designs. The $95 \%$ prediction interval around 0.050 for 500 simulated data sets is indicated in grey $\left(P I_{95 \%}(0.050)=[0.0326 ; 0.0729]\right)$.
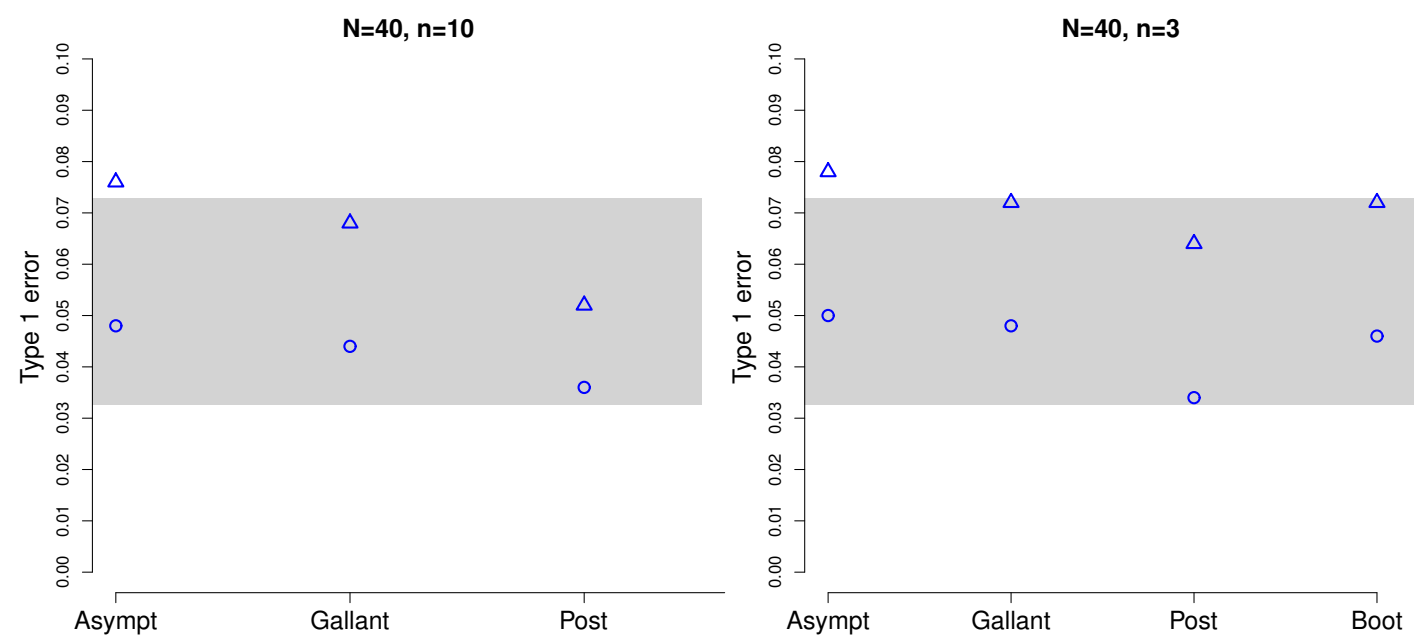

Figure 4: Type I errors of MB-TOST on $\beta_{A U C}^{T r}$ (o) and $\beta_{C \max }^{T r}(\triangle)$ using the different SE calculations on the cross-over rich (left) and sparse (right) designs. The $95 \%$ prediction interval around 0.050 for 500 simulated data sets is indicated in grey $\left(P I_{95 \%}(0.050)=[0.0326 ; 0.0729]\right)$. 
Table II: Estimated power of MB-TOST on $\beta_{A U C}^{T r}$ and $\beta_{C \max }^{T r}$ using the different SE calculation, for the cross-over rich and sparse designs.

\begin{tabular}{|c|c|c||c|c|}
\cline { 2 - 5 } \multicolumn{1}{c|}{} & \multicolumn{2}{c||}{ Rich $(\mathrm{n}=10)$} & \multicolumn{2}{c|}{ Sparse $(\mathrm{n}=3)$} \\
\cline { 2 - 5 } \multicolumn{1}{c|}{} & $\beta_{A U C}^{T r}$ & $\beta_{C \max }^{T r}$ & $\beta_{A U C}^{T r}$ & $\beta_{C \max }^{T r}$ \\
\hline Asympt & 1.000 & 1.000 & 0.998 & 1.000 \\
\hline Gallant & 1.000 & 1.000 & 0.998 & 1.000 \\
\hline Post & 0.988 & 0.998 & 0.996 & 0.996 \\
\hline
\end{tabular}



